\title{
PRINCIPAIS BENEFÍCIOS DA FISIOTERAPIA NO AUMENTO DA PERFORMANCE DOS ATLETAS DE ELITE
}

\author{
Winisthon Wanner de Souza Cabral ${ }^{1}$ \\ Kauara Vilarinho Santana Vieira ${ }^{2}$
}

RESUMO: Este trabalho tem como objetivo analisar a importância da fisioterapia e sua influência na performance dos atletas de elite. Com tudo isso, a fisioterapia tem sido um dos métodos mais recomendados e utilizados na prevenção e tratamento da performance dos atletas de elite, sendo assim são aplicadas sessões de exercícios cinesioterapêuticos para promover: a melhora da mobilidade articular, fortalecimento, flexibilidade, manutenção e ganho de equilíbrio e precisão na coordenação motora na performance dos atletas de elite. Diante disso, para a elaboração deste artigo realizou-se uma pesquisa bibliográfica, baseando-se nas ideias de diferentes autores, através da técnica de revisão de literatura, sendo utilizados artigos relacionados ao tema em questão, em língua inglesa e portuguesa, e consulta nas bases de dados PubMed-Medline da United States National Library of Medicine, National Institutes of Health e Scielo - Scientific Electronic Library Online e Google Acadêmico, separando os artigos de 2011 até 2021. Conclui-se que, a atuação do Fisioterapeuta Esportivo é encontrar os melhores exercícios e as práticas para cada desportista, levando-os para atividades que potencializem o seu desempenho, apoiando na prevenção de lesões nos atletas de elite.

Palavras-chave: Atletas e elite. Alta performance. Atuação Fisioterapêutica.

ABSTRACT: This work aims to analyze the importance of physical therapy and its influence on the performance of elite athletes. With all this, physiotherapy has been one of the most recommended and used methods in the prevention and treatment of the performance of elite athletes, so kinesiotherapeutic exercise sessions are applied to promote: the improvement of joint mobility, strengthening, flexibility, maintenance and gain balance and precision in motor coordination in the performance of elite athletes. Therefore, for the preparation of this article, a bibliographical research was carried out, based on the ideas of different authors, through the literature review technique, using articles related to the topic in question, in English and Portuguese, and consultation in the PubMed-Medline databases of the United States National Library of Medicine, National Institutes of Health and Scielo - Scientific Electronic Library Online and Academic Google, separating articles from 2011 to 202I. It is concluded that the role of the Sports

\footnotetext{
${ }^{\mathrm{I}}$ Acadêmico do ro ${ }^{\circ}$ período do Curso de Fisioterapia da Unibras - Faculdade de Rio Verde. E-mail: cjsilva2oo8@faculdadeobjetivo.com.br.

${ }^{2}$ Professora do Curso de Fisioterapia da Unibras- Faculdade de Rio Verde e orientadora. da pesquisa. E-mail: maria.santos@faculdadeobjetivo.com.br.
} 
Physiotherapist is to find the best exercises and practices for every athlete, taking them to activities that enhance their performance, supporting the prevention of injuries in elite athletes.

Keywords: Athletes and Elite. Performance. Physiotherapeutic Performance.

\section{INTRODUÇÃO}

Este trabalho trata sobre a importância da fisioterapia e a sua influência na performance dos atletas de elite, tendo como área de concentração fisioterapia desportiva. Assim se quer responder ao seguinte questionamento com esta pesquisa: de que forma a fisioterapia pode influenciar na performance dos atletas de elite prevenindo lesões e ampliando sua atuação? (GAYA, 2015).

Este artigo se justifica em analisar a importância da fisioterapia e sua influência na performance dos atletas de elite. Assim, esta pesquisa trata sobre a importância da fisioterapia na performance dos atletas de elite, sendo necessária uma maior atenção para os ligamentos e os músculos, que são as maiores fontes de trabalho destes profissionais. Sabe-se que cada esporte tem seu nível de complexidade e características de: espaço, tempo, dinâmica, exigências físicas e psicológicas. Um desempenho eficaz de um atleta requer um bom funcionamento do seu sistema orgânico (LOPES, 2016).

Como objetivo geral se quer demonstrar a importância da fisioterapia na performance dos atletas de elite e como objetivos específicos: avaliar à aplicação da fisioterapia e como esta pode influenciar na performance dos atletas de elite; analisar e definir a melhor aplicabilidade da fisioterapia na performance dos atletas de elite; descrever a fisioterapia e como esta ajuda na performance dos atletas de elite e apresentar as reais funções da fisioterapia traumato-ortopédica e sua contribuição para a performance dos atletas de elite (COURY, 2019).

\section{REFERENCIAL TEÓRICO}

\section{I Conceito de Atletas de Alta Performance e da Fisioterapia Esportiva}

Ao se falar sobre altetas alta de performance constata-se que este é sinônimo de preparo e condicionamento físico acima de qualquer média, condicionamento físico é fator essencial para o sucesso ou fracasso da atuação deste profissional (PEDRINELLI, 2013). 
Já a fisioterapia esportiva "é uma área da fisioterapia focada em atletas e praticantes de exercícios físicos, sua função é prevenir e proteger o corpo e tratar fraturas ou lesões" (CRUZ, 2020, p.oI). Assim, a grande missão do fisioterapeuta que cuida de atletas é: “ajudá-los a alcançar seu mais alto desempenho sem se prejudicar. Em caso de problemas, permitir que se recuperem quanto antes e retornem a seu desempenho padrão" (CRUZ, 2020, p.02).

"O treinamento de atletas de elites se torna mais exaustivo e a dieta se torna mais rigorosa, refeições se tornam menos calóricas, perder peso e secar é penoso, o atleta passa fome, mas acaba se acostumando com isso depois de alguns dias, acrescenta mais aerobiose ao programa de treino, muitas vezes acorda mais cedo para encarar uma bicicleta ou correr na rua em jejum" (GUIMARÃES, 2or6, p.I).

Constata-se que: "sem um acompanhamento adequado o desgaste físico pode se tornar um problema grave e a sobrecarga constante pode levar a sérias complicações" (CRUZ, 2020, p. oI).

\subsection{Performance de atletas de elite}

Os atletas de alto-rendimento apesar de terem em sua amplitude acesso a boa alimentação, boa moradia, educação de qualidade, acesso a serviços de saúde com tratamento especializado, a atividade de lazer e uma boa posição econômica, os fatores destrutivos acabam superando o conjunto dos fatores protetores (SIMÕES, 2015).

Existem diversos métodos e estratégias para serem utilizados na fase précompetição de um atleta de alta performance, dentre as estratégias mais utilizadas destacam-se o déficit calórico e a inclusão de trabalho aeróbico na rotina do atleta (RESENDE,2014)

Em se tratando do tema em baila, constata-se que este é muito polêmico por ainda requer em alguns casos um pouco de mais de dedicação do atleta e principalmente, que às vezes retém líquidos no corpo, o que pode prejudicar o seu rendimento, assim como as lesões (PASTRE, 2015). 


\subsection{Principais Disfunções Neuromusculares em Atletas de Elite}

Como a principal lesão em atletas de elites cita-se assim, por exemplo, os ligamentos e os músculos do tornozelo e do pé sendo projetados para promoverem estabilidade bem como, mobilidade às estruturas distais do membro inferior (KISNER e COLBY, 2005, citados por MATOS, 2012, p.oI)

Assim, cita-se ainda Hall: "O tornozelo contém um total de 26 ossos e numerosas articulações. Juntos, os ossos e articulações do pé fornecem uma base de suporte para o corpo ereto e o auxiliam na adaptação em terrenos irregulares e na absorção de impactos" (HALL, 2019, p.oI).

A região do tornozelo inclui as articulações: tibiofibular distal, tibiotalar e fibutalar. A articulação é sustentada pelos ligamentos tibiofibular anterior e posterior e pelo ligamento tibiofibular interósseo (HALL, 2019).

Quando se trata da articulação do tornozelo este, infelizmente carrega toda a força do corpo, coloca-se segundo Dutton (20I6), a articulação do tornozelo sustentando maior carga por área, do que qualquer articulação do corpo.

Assim, também tem disfunções na coluna que é quando não existe uma coordenação, bem-feita quando está em apoio monopodal suportando todo o peso do corpo, que aumenta pela energia cinética, durante a marcha, na corrida ou na preparação para o salto (COURY, 2019).

Estruturas do tornozelo Kisner e Colby (2015), falam que os ligamentos, e os músculos do tornozelo e do pé são projetados para promover estabilidade bem como mobilidade às estruturas distais do membro inferior. Hall (2019), o tornozelo contém um total de 26 ossos e numerosas articulações. Juntos, os ossos e articulações do pé fornecem uma base de suporte para o corpo ereto e o auxiliam na adaptação em terrenos irregulares e na absorção de impactos.

Com tudo isso, contata-se que, com o grande avanço tecnológico, o que possibilita maior qualidade de vida e saúde, este avanço traz consigo um meio de cuidar melhor do aparelho muscular dos atletas de elite, sendo que a fisioterapia se torna essencial neste contexto (PAZIN, 2018). 


\subsection{Fisioterapia Preventiva em Atletas de Elite}

Em se tratando, do tema desta pesquisa, sobre a importância da fisioterapia na performance dos atletas de elite, constata-se que a pré-competição é um momento em que muitos atletas de elite realizam algumas mudanças em seus protocolos de treino e dieta, para conseguirem mostrarem-se bem em sua atuação, sendo papel do fisioterapeuta verificar se essas mudanças são benéficas para o atleta (GAYA,20ı6).

Nesta fase os atletas criam um déficit calórico, para que ocorra uma maior perca de gordura corporal. Apesar de nesta etapa os atletas estarem ingerindo pouca caloria, a intensidade do treino tem que ser mantida a todo custo para preservar a massa muscular, que está sendo prejudicada pela dieta restrita e rigorosa, assim, com esta situação de précompetição, que se verifica a real necessidade de uma Fisioterapia Preventiva (PANNAIN, 2012).

Diante disso, ressalta-se a importância da prevenção, que também é parte da responsabilidade dos fisioterapeutas, deste modo, o trabalho fisioterapêutico é: "garantir que o atleta trabalhe dentro de seus próprios limites, protegendo seu sistema locomotor de excessos, desequilíbrios, tratando de: luxações, fraturas, distensões, entorses, tendinites, contusões, lesões ligamentares, abrasões, cortes, bolhas e calos” (CRUZ, 2020, p.or).

\subsection{Abordagens fisioterapêuticas para o Atleta de Alta performance}

Duas pessoas nunca são iguais e, raramente, treinam da mesma maneira. Cada uma é diferente, em sua genética, experiências atléticas anteriores, hábitos alimentares, metabolismo, objetivos e potencial de adaptação, deste modo, é que se pode constatar a importância da fisioterapia no aumento da performance dos atletas de elites. Por todas estas razões, os altetas de elite devem possuir programas individuais de treinamento, considerando o seu nível de desenvolvimento (BOMPA, 20II, p.23). Dentre os principais métodos de treinamento abordagens fisioterapêuticas no aumento da performance de atletas de elite cita-se:

- A Pirâmide crescente: de acordo com Guimarães (2012), a primeira série servirá de aquecimento, nesta série devemos utilizar uma carga mais leve para que o atleta consiga realizar em torno de 15 repetições, na segunda série devemos aumentar a carga para fazer em torno de 12 repetições, na terceira série usaremos uma carga para fazer em torno de Io 
repetições, na quarta usaremos uma carga para fazer em torno de 8 repetições e na quinta série usaremos uma carga alta pois o atleta já está bem aquecido e assim o risco de lesão se torna menor, nesta série o atleta deverá realizará em torno de 6 repetições máxima.

- A Pirâmide decrescente: aqui acontece o contrário da pirâmide crescente, onde a carga diminui a cada série e as repetições aumentam proporcionalmente (BOMPA, 20II).

- A Pirâmide dupla: esta representa duas pirâmides, onde primeiramente realizamos a pirâmide crescente e após isto realizamos a pirâmide decrescente (BOMPA, 20II). De acordo com o autor este método promove mais benefícios ao treinamento do que as pirâmides citadas anteriormente, pois provoca melhores adaptações neuromusculares.

- O Drop set: este é um método onde o atleta de realizar a primeira série até a falha muscular, quando então a carga será diminuída (em torno de $40 \%$ ); a série será iniciada imediatamente até que o atleta atinja a falha total (GUIMARÃES, 2012).

- Método do Super-Set: este método foi criado em 1950, onde em sua forma tradicional executa-se um exercício para um determinado grupo muscular e sem dar intervalo executa-se um exercício para a musculatura antagonista do primeiro exercício (PEREIRA, 2017).

\subsection{Benefícios Funcionais que o Atleta de Alta performance apresenta com o trabalho do Fisioterapeuta}

Ao se falar sobre do tema deste trabalho, sobre a importância da fisioterapia na performance dos atletas de elite, verifica-se que esta é de suma importância para que não ocorram acidentes nos músculos, ligamentos, dentre outras partes de corpo dos esportistas, principalmente em época de competição, sendo o fisioterapeuta essencial neste quesito (COURY, 2019).

As intensas atividades físicas durante as competições e treinamentos sobrecarregam o sistema musculoesquelético, exigindo do corpo níveis de força muscular, transferências de pesos e amplitudes articulares, muito acima que os níveis fisiológicos para que possam suportar as mudanças de direção, interrupções abruptas e de grande impacto. Os conhecimentos: anatômicos, fisiológicos, cinesiológicos e biomecânicosdo do fisioterapeuta são imprescindíveis para que o atleta de elite tenha um preparo funcional capaz de suportar todas essas forças e adversidades do esporte (PAZIN, 2018). 
Com tudo isso, a atuação do Fisioterapeuta Esportivo também é encontrar os melhores exercícios e as práticas para cada desportista, levando-os para prática de atividades que potencializem o seu desempenho, apoiando na prevenção de lesões. (SIMÕES, 2015).

Com tudo isso esta pesquisa tratou sobre a importância da fisioterapia na performance dos atletas de elite, sendo necessário uma maior atenção para a prevenção de lesões, sendo são as maiores fontes de trabalho destes profissionais e casos estas ocorram, que seja feito tratamento por profissional habilitado (STRYER, 2018).

\section{METODOLOGIA}

Para a elaboração deste artigo realizou-se uma pesquisa bibliográfica, através da técnica de revisão de literatura, sendo utilizados artigos relacionados ao tema em questão, em língua inglesa e portuguesa, e consulta nas bases de dados PubMed-Medline da United States National Library of Medicine, National Institutes of Health e Scielo - Scientific Electronic Library Online e Google Acadêmico, separando os artigos de 20II até 202I. Sendo que os descritores/palavras-chave utilizados nesta pesquisa são: fisioterapia desportiva, atletas de elite, período pré-competição, prevenção de lesões e atuação do fisioterapeuta.

Neste trabalho será utilizada a pesquisa bibliográfica como base para a busca de dados. Deste modo, Lakatos e Marconi (1991, p. 73), afirmam que a pesquisa bibliográfica é: "Abrange toda bibliografia já tornada pública em relação ao tema em estudo, desde publicações avulsas, boletins, jornais, revistas, livros, pesquisas, monografias, teses, material cartográfico até meios de comunicação orais, dentre outros. “

Para o desenvolvimento deste trabalho, foram realizadas pesquisas bibliográficas intentando construir um referencial teórico capaz de possibilitar o entendimento do tema, para que possam sanar as hipóteses já elencadas e as que surgirão através do estudo (FERNANDES, 2000).

Espera-se que, a abordagem sobre este assunto gere um conhecimento aprimorado, sane dúvidas e respondam as hipóteses elencadas, nesta pesquisa. 


\section{CONSIDERAÇÕES FINAIS}

Através da pesquisa realizada constatou-se que, a atuação do Fisioterapeuta Esportivo também é encontrar os melhores exercícios e as práticas para cada desportista, levando-os para prática de atividades que potencializem o seu desempenho, apoiando na prevenção de lesões.

Em se tratando do tema em baila, constata-se que este é muito polêmico por ainda requer em alguns casos um pouco de mais de dedicação do atleta e o que pode prejudicar o seu rendimento.

Depreende-se que, esta pesquisa tratou sobre a importância da fisioterapia na performance dos atletas de elite, sendo necessário uma maior atenção para a prevenção de lesões, sendo são as maiores fontes de trabalho destes profissionais e casos estas ocorram, que seja feito tratamento por profissional habilitado

\section{REFERÊNCIAS}

ARAÚJO. 2015. O momento pré-competição para atletas de elite. Disponível em: In:http://www.bodybuilding.com/fun/teenryanswan7.htm. Acesso em: i7 de junho de 202I.

BOMPA, T. O., CORNACHIA, L. J. Treinamento de força consciente. Porto Alegre. Phorte, 2010.

COURY, H. J. Prevenção de lesões músculo-esqueléticas ocupacionais: abordagem preventiva da fisioterapia. Fisioterapia e Pesquisa, v. 6, p. 24-24, 2019.

CRUZ, Diene Oliveira. Fisioterapia para atletas: importância, benefícios e técnicas.2020. Disponível em: https://www.hong.com.br/fisioterapia-para-atletas-importanciabeneficios-e-tecnicas/Acesso em: Io de outubro de 202I.

DUTTON, Mark. Fisioterapia Ortopédica: Exame, Avaliação e Intervenção. São Paulo: Artmed, 2016.

FERNANDES, José. Técnicas de Estudo e Pesquisa. 2. ed. Goiânia: Kelps, 2000. 
FLECK, Paula. Fundamentos do Treinamento de Força Muscular. Porto Alegre: Artmed, 2010.

GAYA A. et al. Talento esportivo: estudo de indicadores somato-motores na seleção para - desporto de excelência. 2015. Disponível em: http://portal.esporte.gov.br/arquivos/snear/talento_esportiv o/base_teorica_talento_esportivo_desporto_excelencia.pdf . Acesso em: io de outubro de 2021.

GAYA, A. Projeto Esporte Brasil, Ministério do Esporte. 2016. Disponível em: http://portal.esporte.gov.br/arquivos/snear/talento_esportiv o/proesp_br.pdf. Acesso em: Io de outubro de 2021.

GIL, A. C. Como elaborar projetos de pesquisa. 3. ed. São Paulo: Atlas, I994.

GLANER, M.F. Tópicos especiais em antropometria. Revista Mineira de Educação Física, Viçosa, v. I2, n. 2, p. 143-158, 2016 GUEDES, D. Manual prático para avaliação em educação física. Barueri SP: Editora Manole Ltda, 2006.

GUIMARÃES, N. ET AL. 2016. Métodos Diuréticos: Retido é o Senhor seu Alvo. Disponível em: In:http://www.hipertrofia.org/forum/topic/22613-falando-depr\% $\mathrm{C}_{3} \% \mathrm{~A}_{9}$-contestmetodos-diur\% $\mathrm{C}_{3} \%$ A9ticos. Acesso em: I7 de junho de 2021.

GUIMARÃES, N., MARQUES, W. Manifesto anabólico XIX. 2012 Disponível em: In:http://www.w aldemarguimaraes.com.br/news.php?i=2012. Acesso em: 17 de junho de 2021.

HALL, Susan J. Biomecânica básica; [tradução Patrícia Neumam]. - 5. Ed. - Barueri, SP. Manole, 2019. 
JAMES R., Andrews; GARY L., Harrelson; KEVIN E., Wilk. Reabilitação Física das Lesões Desportivas. São Paulo: Guanabara Koogan, 2010.

LAKATOS, E. M.; MARCONI, M. A.: Fundamentos de Metodologia Científica. São

LOPES V.; MAIA J. Períodos críticos ou sensíveis: revisitar um tema polêmico à luz da investigação empírica. Rev Paul Educ Física São Paulo, v. I4, n. 2, p. I28-40, 2016.

MATOS, Rosangela dos Santos. Reabilitação da entorse de tornozelo, 2012. Disponível em: https://portalbiocursos.com.br/ohs/data/docs/34/262__ReabilitaYYo_Entorse_de_tornozelo.pdf. Acesso em: I2 de Junho de 202I.

MENEZES, Estera Muszat; SILVA, Edna Lúcia da. Metodologia da pesquisa e elaboração de dissertação. Florianópolis, 2000.

MORAIS, Raquel Alves. Entorse de Tornozelo e seu tratamento: revisão de literatura. 2018.

http://publicacoesacademicas.unicatolicaquixada.edu.br/index.php/mostrafisioterapia/art icle/view/28I4. Acesso em: I2 de Junho de 2021.

PANNAIN, R. What do You Need to Know to Survive the Judging. 2012 Disponível em: In:http://ricardopannain.blogspot.com.br/2012_O2_ol_archive.html. Acesso em: I7 de junho de 202I.

PASTRE, C. M. Lesões desportivas na elite do atletismo brasileiro: estudo a partir de morbidade referida. Rev Bras Med Esporte, Presidente Prudente, v.II, n.I, p.43-47, 2015.

PAZIN, J. et al. Corredores de rua: características demográficas, treinamento e prevalência de lesões. Revista Brasileira de Cineantropometria e Desempenho Humano. v. Io, n. 3, p. 277-282, 2018 . 
PEDRINELLI, A.; Estudo epidemiológico das lesões no futebol profissional durante a Copa América de 201r. Argentina. Rev Bras Ortop, São Paulo, v.48, n.2, p.131-136, 2013.

PEREIRA, D. S. Avaliação do perfil de estilo de vida de usuários que praticam exercício físico regular comparado a usuários sedentários inseridos em uma estratégia de saúde da família - Região sudeste do Brasil. Revista APS, São Paulo, v. 20, n. I, p. 30-39, 2017.

RESENDE, M.M.; Fisioterapia e prevenção de lesões esportivas. Fisioterapia Brasil, Belém, v.15, n.3, p.219-223, 2014.

SCHWARZENEGGER, A. Enciclopédia da musculação. Tradução de Márcia dos Santos Dornelles e Jussara Burnier. 2. EdPorto Alegre: Artmed, 20or.

SIMÕES, N. V. N. Lesões desportivas em praticantes de atividade física: Uma revisão bibliográfica. Rev. bras. Fisioter., v. 9, n. 2, p.123-128, 2015.

STOPPANL, 2014. J. Is Fasted Cardio The Best For Burning Fat. In: Disponível em: http://www.bodybuilding.com/fun/is-fasted-cardio-the-best-for-burning-fat.html. Acesso em: i7 de junho de 2021.

STRYER, Lucas. Adaptações Cardiovasculares ao Exercício. São Paulo: Artmed, 2018. 